\title{
Galleria mellonella as an Infection Model for Bacillus anthracis Sterne
}

\author{
Jacob A. Malmquist ${ }^{\dagger}$, Madison R. Rogan ${ }^{\dagger}$ and Shauna M. McGillivray* \\ Department of Biology, Texas Christian University, Fort Worth, TX, United States
}

Understanding bacterial virulence provides insight into the molecular basis behind infection and could identify new drug targets. However, assessing potential virulence determinants relies on testing in an animal model. The mouse is a well-validated model but it is constrained by the ethical and logistical challenges of using vertebrate animals. Recently the larva of the greater wax moth Galleria mellonella has been explored as a possible infection model for a number of pathogens. In this study, we developed

OPEN ACCESS

Edited by:

Eric Ghigo,

IHU Mediterranee Infection, France

Reviewed by:

Rodnei Dennis Rossoni, São Paulo State University, Brazil Siouxsie Wiles,

The University of Auckland, New Zealand

*Correspondence:

Shauna M. McGillivray

s.mcgillivray@tcu.edu

${ }^{\dagger}$ Present address:

Jacob A. Malmquist,

Long School of Medicine, San

Antonio, TX, United States

Madison R. Rogan,

Department of Pathology, University of Texas Medical Branch, Galveston, TX,

United States

Specialty section:

This article was submitted to

Bacteria and Host,

a section of the journal

Frontiers in Cellular and Infection

Microbiology

Received: 09 August 2019 Accepted: 04 October 2019 Published: 18 October 2019

Citation:

Malmquist JA, Rogan MR and McGillivray SM (2019)

Galleria mellonella as an Infection Model for Bacillus anthracis Sterne. Front. Cell. Infect. Microbiol. 9:360. doi: 10.3389/fcimb.2019.00360 G. mellonella as an infection model for Bacillus anthracis Sterne. We first validated two different infection assays, a survival assay and a competition assay, using mutants containing disruptions in known B. anthracis virulence genes. We next tested the utility of $G$. mellonella to assess the virulence of transposon mutants with unknown mutations that had increased susceptibility to hydrogen peroxide in in vitro assays. One of these transposon mutants also displayed significantly decreased virulence in G. mellonella. Further investigation revealed that this mutant had a disruption in the petrobactin biosynthesis operon (asbABCDEF), which has been previously implicated in both virulence and defense against oxidative stress. We conclude that $G$. mellonella can detect attenuated virulence of $B$. anthracis Sterne in a manner consistent with that of mammalian infection models. Therefore, G. mellonella could serve as a useful alternative to vertebrate testing, especially for early assessments of potential virulence genes when use of a mammalian model may not be ethical or practical.

Keywords: Galleria mellonella, Bacillus anthracis, Sterne, infection model, hydrogen peroxide, virulence

\section{INTRODUCTION}

Bacillus anthracis is a gram-positive, spore-forming bacterium that causes the deadly disease anthrax. Major virulence determinants include the lethal and edema toxins encoded on the pXO1 plasmid and the capsule encoded on the pXO2 plasmid. Additionally, there are putative virulence genes encoded within the chromosome (Read et al., 2003) and a number of groups have experimentally demonstrated that chromosomally encoded genes are essential for virulence (Gat et al., 2005; McGillivray et al., 2009; Kern and Schneewind, 2010; Chitlaru et al., 2011; Jenkins et al., 2011). The Sterne strain of $B$. anthracis lacks the $\mathrm{pXO} 2$ plasmid $\left(\mathrm{pXO}^{+}, \mathrm{pXO}^{-}\right)$and is avirulent in humans and normal mice due to increased sensitivity to complement, but it is a well-established alternative to the fully virulent $B$. anthracis $\left(\mathrm{pXO}^{+}, \mathrm{pXO}^{+}\right)$strains which can only be studied in a limited number of labs (Harvill et al., 2005). Complement-deficient mice (A/J, DBA/J) are commonly used as the model system for the Sterne strain (Welkos and Friedlander, 1988), but there are ethical and logistical constraints to using any vertebrate model. This is particularly true in preliminary studies for which it is difficult to justify the use of large numbers of vertebrate animals. While in vitro and cell-based assays have their place, an animal model is eventually needed for in vivo infection studies. 
Invertebrate models have shown promise as infection models. They are inexpensive and easy to maintain, and they possess conserved innate immune system components (Glavis-Bloom et al., 2012). C. elegans has previously been used with B. anthracis Sterne as well as other bacterial pathogens (Kho et al., 2011; Glavis-Bloom et al., 2012; Franks et al., 2014), but there are limitations to this model. C. elegans cannot be incubated at $37^{\circ} \mathrm{C}$, the optimal temperature for bacterial pathogens, and the route of infection is via ingestion which is convenient, but can lead to uneven exposure to pathogen. It also does not seem to possess phagocytic cells analogous to macrophages or neutrophils (Gravato-Nobre and Hodgkin, 2005). Moreover, infection of $C$. elegans by $B$. anthracis Sterne also requires additional stresses to be placed on the worm including exposure to the Cry5B toxin and incubation at abnormally high temperatures for the worm (Kho et al., 2011; Franks et al., 2014). The larva of the greater wax moth Galleria mellonella has recently emerged as a desirable infection model with several advantageous physical and molecular features. These include a size sufficient for direct injection; the ability to incubate the larvae at $37^{\circ} \mathrm{C}$; and the presence of innate immune defenses that are analogous to mammalian defenses including phagocytic hemocytes, the production of reactive oxygen species (ROS), opsonizing proteins that recognize conserved microbial patterns, and the production of antimicrobial peptides (AMPs) such as lysozyme (Kavanagh and Reeves, 2004; Tsai et al., 2016). G. mellonella has been used as an infection model for both gram-positive and gram-negative bacteria including Staphylococcus aureus, Streptococcus pyogenes, Enterococcus faecalis, Pseudomonas aeruginosa, Escherichia coli, and Acinetobacter baumannii to name a few. In vivo studies utilizing $G$. mellonella have been used to both identify new virulence factors and evaluate potential antibacterial compounds (Tsai et al., 2016).

In this study, we developed $G$. mellonella survival and competition assays for use with $B$. anthracis Sterne and validated these infection models using mutants with disruptions in previously identified virulence genes of $B$. anthracis. We then conducted a small proof-of-concept screen with transposon mutants to demonstrate the utility of $G$. mellonella for studying potential virulence determinants of $B$. anthracis Sterne. We find that $G$. mellonella is a suitable animal model for B. anthracis Sterne and that this could be a practical and ethical manner in which to assess virulence.

\section{METHODS}

\section{Bacterial Growth Conditions and Strains}

Bacillus anthracis Sterne strain $34 \mathrm{~F} 2\left(\mathrm{pX01}{ }^{+}, \mathrm{pX02}^{-}\right)$was cultured in Brain-Heart Infusion (BHI) media (Hardy Diagnostics, Santa Maria CA, USA) at $37^{\circ} \mathrm{C}$ under aerobic conditions. Antibiotics were used at the following concentrations: E. coli: erythromycin $500 \mu \mathrm{g} / \mathrm{ml}$ (Erm500); B. anthracis: erythromycin $5 \mu \mathrm{g} / \mathrm{ml}$ (Erm5) and kanamycin $50 \mu \mathrm{g} / \mathrm{ml}$ (Kan50) (all Sigma, St. Louis MO, USA). Construction of $B$. anthracis strains $\Delta \mathrm{pXO}, \Delta c l p X, \Delta y c e G H$ and the transposon library were previously described (van Sorge et al.,
TABLE 1 | Primers and oligonucleotides used in this study.

\begin{tabular}{|c|c|}
\hline Name & Sequence \\
\hline purH Fwd-Xhol & $\begin{array}{l}\text { 5'-ACAGTCTCGAGAAAGAGAACGGTGAAGTAGCAGAG- } \\
3^{\prime}\end{array}$ \\
\hline purH Rev-HindllI & 5' -GACTAAGCTTCATGAATATCCGTACCTACTCCAACA-3' \\
\hline mntA Fwd-Xhol & $\begin{array}{l}\text { 5'-ACAGTCTCGAGAACCCGCATGAATATGATCCACTAC- } \\
3^{\prime}\end{array}$ \\
\hline mntA Rev-HindllI & 5'-GACTAAGCTTCGTITCTCCTCAGGGATTGATG-3' \\
\hline htrA Fwd-EcoRl & 5'-ACGTCTCGAGTGCAATGCAACCGACAGG-3' \\
\hline htrA Rev-Xhol & 5'-tacgGAATTCGCACGCTTATCGCCATCGA-3' \\
\hline pHY304 Fwd & 5'-ACGACTCACTATAGGGCGAATTGG-3' \\
\hline$\Delta$ purH confirm Rev & 5'-GCTTGCGCTTGTCGCTI-3' \\
\hline$\Delta$ mntA confirm Rev & 5'-AGGTAATGGGATITGGGAAGGTG-3' \\
\hline$\Delta$ htrA confirm Rev & 5'-AACGACACCTACTCCAAGAGC-3' \\
\hline Linker 1 & $\begin{array}{l}\text { 5'-TाTCTGCTCGAATTCAAGCTTCTAACGATGTAC } \\
\text { GGGGACACATG }\end{array}$ \\
\hline Linker 2 & $\begin{array}{l}\text { 5'-TGTCCCCGTACATCGTTAGAACTACTCGTACCA } \\
\text { TCCACAT-3' }\end{array}$ \\
\hline Himar 1-2 Fwd & 5'-GGGAATCATITGAAGGTTGGTACT-3' \\
\hline Y-linker Rev & 5'-CTGCTCGAATTCAAGCTTCT-3' \\
\hline Himar 1-4 Fwd & 5'-TATGCATTTAATACTAGCGAC-3' \\
\hline 4D5 Tn confirm Rev & 5'-TCGCCTCTTGCACACCTTCC-3' \\
\hline
\end{tabular}

2008; McGillivray et al., 2009; Franks et al., 2014). The remaining strains were constructed in this study.

\section{Construction of $\Delta p u r H, \Delta m n t A$, and $\Delta h t r A$ Virulence Mutants}

To disrupt the purH, mntA, and htrA genes by insertional mutagenesis, an internal fragment of each gene was amplified via PCR using primers engineered with $5^{\prime}$ extensions containing restriction sites (described in Table 1). Each amplicon was digested and ligated into the temperature-sensitive plasmid pHY304 (all enzymes NEB, Ipswich, MA, USA), transformed into the E. coli strain MC1061 (Lucigen, Middleton, WI, USA), and plated under Erm500 selection at $30^{\circ} \mathrm{C}$. Plasmids were then extracted via miniprep (IBI Scientific, Peosta IA, USA), transformed into methylation-deficient E. coli strain GM2163, and plated on Erm500 selection. GM2163 grown plasmids were extracted, transformed into electrocompetent B. anthracis Sterne as previously described (Koehler et al., 1994), and plated under Erm5 selection at $30^{\circ} \mathrm{C}$. B. anthracis Sterne containing the insertional mutant plasmid were passed two times at $37^{\circ} \mathrm{C}$ in $\mathrm{BHI}-\mathrm{Erm} 5$ to force plasmid integration. Integration into the bacterial chromosome was confirmed by PCR using the pHY304Fwd primer and the gene-specific confirmation primer located downstream of the original amplicon (Table 1).

\section{Validation of in vitro Phenotypes of $\Delta p u r H$, $\Delta m n t A$, and $\Delta h t r A$}

$\Delta$ purH: Overnight parental and $\Delta p u r H$ cultures were washed and resuspended in PBS then diluted in minimal R-medium (Ristroph and Ivins, 1983) and BHI at a 1:15 dilution. Cultures were incubated at $37^{\circ} \mathrm{C}$ under shaking conditions. The optical 
density (OD) at wavelength $600 \mathrm{~nm}$ was determined after $24 \mathrm{~h}$ to quantify bacterial growth. $\Delta \boldsymbol{m} \boldsymbol{n} \boldsymbol{t} A$ : Parental and $\Delta m n t A$ cultures were grown overnight and then diluted to final concentration of 1:50 in BHI containing 0 or $0.005 \% \mathrm{H}_{2} \mathrm{O}_{2}$ in 96 -well plates. Plates were incubated at $37^{\circ} \mathrm{C}$ under static conditions for $24 \mathrm{~h}$ and growth assessed by measuring OD. $\Delta \boldsymbol{h t r} \boldsymbol{A} \mathrm{H}_{2} \mathrm{O}_{2}$ : Parental and $\triangle h t r A$ cultures were grown to $\log$ phase $(\mathrm{OD}=0.4)$ and then diluted 1:20 in BHI containing 0 or $0.0025 \% \mathrm{H}_{2} \mathrm{O}_{2}$ in 96-well plates. Plates were incubated at $37^{\circ} \mathrm{C}$ under static conditions and growth was assessed at $8 \mathrm{~h}$ by measuring OD. $\boldsymbol{\Delta} \boldsymbol{h t r} \boldsymbol{A}$ temperature: Parental and $\Delta h t r A$ cultures were grown to log phase $(\mathrm{OD}=0.4)$ and then diluted 1:100 in $\mathrm{BHI}$ and grown under shaking conditions at $37^{\circ} \mathrm{C}$ or $44^{\circ} \mathrm{C}$. Growth was monitored and OD measured every hour for $6 \mathrm{~h}$.

\section{G. mellonella Infection Assays}

Galleria mellonella larvae were obtained from Rainbow Mealworms (www.rainbowmealworms.net), stored at $4{ }^{\circ} \mathrm{C}$ to induce torpidity, and used within 5 days of receipt. Only larvae weighing 190-220 mg were used in assays. Injections were done through the posterior cuticle using an automated pump (New Era Pump Systems NE-500, Farmingdale, NY, USA) and a 27-gauge needle.

\section{Survival Assay}

Bacillus anthracis Sterne strains were grown to an OD 0.4 in BHI $\left(\sim 1 \times 10^{7}\right.$ colony forming units $[\mathrm{cfu}]$ per $\left.\mathrm{ml}\right)$, washed and resuspended in PBS, and then diluted prior to injection. For the dose curve in Figure 1A, bacteria were diluted in PBS 1:100, $1: 10$, or used undiluted; for all other survival assays, bacteria were diluted 1:2 in PBS. Ten microliters of diluted bacteria were injected per larva and 10 larvae were injected for each condition. The starting inoculum was confirmed through serial dilution and enumeration of cfu. After injection, larvae were observed at room temperature for $15-30 \mathrm{~min}$ to ensure they recovered from injection and were transferred to an incubator at $37^{\circ} \mathrm{C}$. Surviving larvae were counted at 24,48 , and 72 -h post-injection.

\section{Competition Assay}

Bacillus anthracis Sterne strains were grown to log phase (OD $=0.4$ ) and washed and resuspended in PBS. Mutant strains were mixed with the parental (wild-type) strain at a $1: 1$ ratio. Prior to injection, the mixed culture was plated on both plain and antibiotic-containing media (Erm5 for $\Delta h \operatorname{tr} A$; Kan50 for $\Delta y c e G H)$ to ensure an equal starting ratio. Ten microliters of the mixed culture $\left(\sim 1 \times 10^{5}\right.$ total cfu $)$ was injected into each larva and 5 larvae were injected for each condition. After injection, larvae were observed for $15-30 \mathrm{~min}$ at room temperature to ensure they recovered from injection and then were incubated at $37^{\circ} \mathrm{C}$ for $6 \mathrm{~h}$. After incubation, larvae were briefly rinsed in $70 \%$ ethanol followed by sterile water and transferred to $2 \mathrm{ml} \mathrm{screw-}$ top tubes containing $400 \mu \mathrm{l}$ PBS and $1 \mathrm{~mm}$ ceramic beads (Fox Industries, Fairfield NJ, USA). Larvae were homogenized using the Fast Prep-24 (MP Biomedicals, Solon, OH, USA) with two pulses at $4.5 \mathrm{~m} / \mathrm{s}$ for $45 \mathrm{~s}$. Surviving bacteria were enumerated by serial dilution plating on BHI (total cfu) and BHI-Erm or BHI-Kan media (mutant cfu). Wild-type cfu were determined

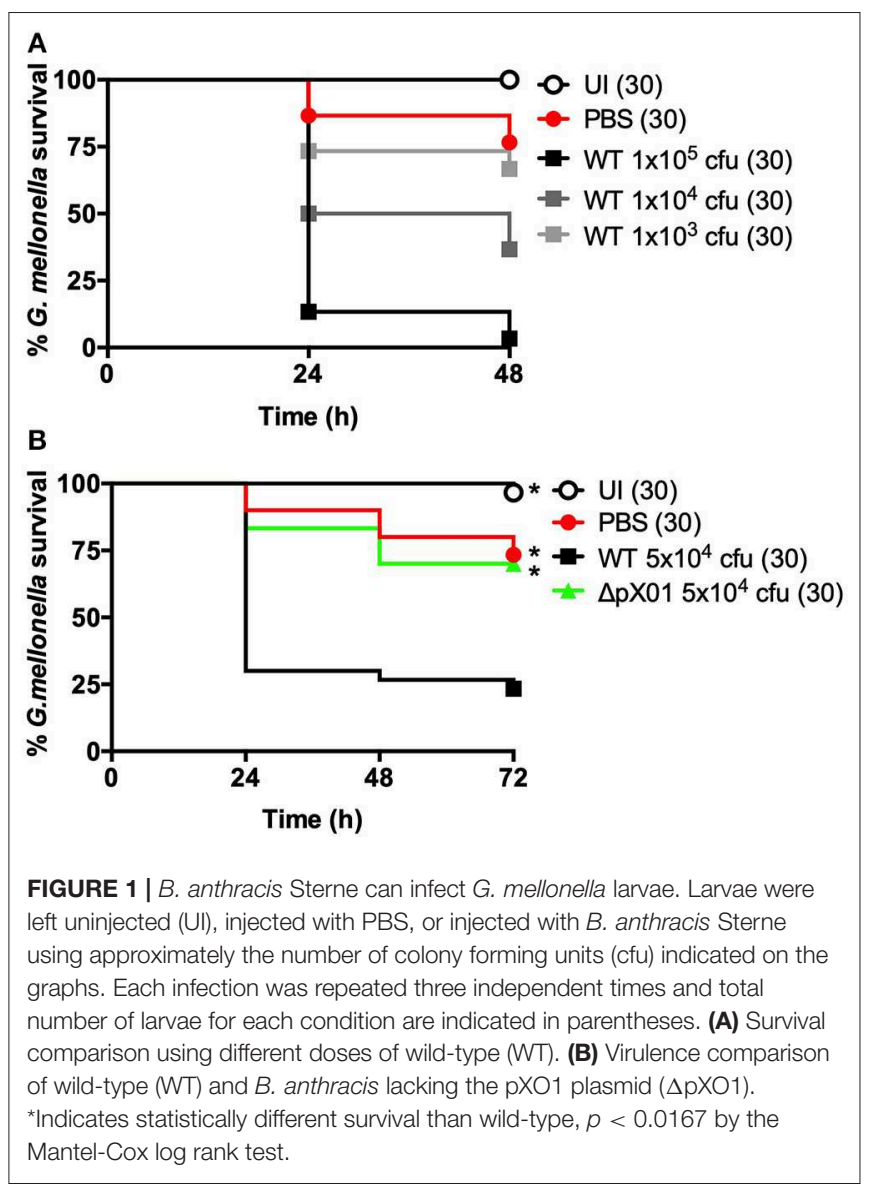

by subtracting the antibiotic-resistant mutant cfu from the total cfu and the percent recovery was calculated by dividing the number of wild-type or mutant cfu by the total number of cfu and multiplying by 100 .

\section{$\mathrm{H}_{2} \mathrm{O}_{2}$ Assays Using Transposon Mutants}

Transposon mutants were grown overnight in 96-well plates and then diluted 1:100 into a new 96-well plate containing $200 \mu \mathrm{l}$ of 0 or $0.01 \% \mathrm{H}_{2} \mathrm{O}_{2}$ in $\mathrm{BHI}$. Plates were incubated overnight at $37^{\circ} \mathrm{C}$ under static conditions and growth was measured at OD 0.4.

\section{Identification of Site of Transposon Insertion}

The site of transposon insertion was identified using the Y-linker method (Kwon and Ricke, 2000). Briefly, $18 \mu \mathrm{l}$ of the linker 2 oligonucleotide $(100 \mu \mathrm{M})$ was phosphorylated with PNK $(2 \mu \mathrm{l}$ enzyme, $4 \mu \mathrm{l} 10 \mathrm{x}$ T4 ligase buffer, $16 \mu \mathrm{l} \mathrm{H}_{2} \mathrm{O}$ ) at $37^{\circ} \mathrm{C}$ for $1 \mathrm{~h}$ and then denatured at $65^{\circ} \mathrm{C}$ for $20 \mathrm{~min}$. To form the Y-linker, $18 \mu \mathrm{l}$ of linker 1 oligonucleotide $(100 \mu \mathrm{M})$ was then added to the mixture and it was incubated at $95^{\circ} \mathrm{C}$ for $5 \mathrm{~min}$ before being slowly cooled to room temperature to allow for linker 1 and 2 to anneal. Genomic DNA was extracted using phenol/chloroform and $5 \mu \mathrm{g}$ was digested with NlaIII. Two-hundred nanograms of digested DNA was ligated to $5 \mu \mathrm{l}$ of the annealed Y-linker using $1 \mu \mathrm{l}$ T4 ligase in a $20 \mu \mathrm{l}$ total reaction (all enzymes NEB, Ipswich, 
MA, USA). The ligation was diluted to a final volume of $200 \mu \mathrm{l}$ and then heat denatured, and $2 \mu \mathrm{l}$ was used for template in a PCR reaction using the Y-linker primer and the transposon specific primer Himar 1-2. PCR products were purified and sent for sequencing using the Himar 1-4 primer. Results were BLASTed against the $B$. anthracis genome and the site of insertion was confirmed using the Himar 1-2 primer and a primer located downstream of the insertion site. All primers/oligonucleotides are listed in Table $\mathbf{1 .}$

\section{Statistics}

GraphPad Prism (San Diego, CA, USA) was used for all statistical analysis. To correct for error caused by multiple comparisons in the survival curves, the level of statistical significance was set as 0.05 divided by the number of comparisons being made.

\section{RESULTS}

\section{Survival Assay Using G. mellonella}

Our first question was whether B. anthracis Sterne could reliably infect G. mellonella. To test this, we adapted a previously described protocol (Ramarao et al., 2012) using G. mellonella ordered from an online bait store (Rainbow Mealworms). We selected G. mellonella larvae weighing between 190 and $220 \mathrm{mg}$ in order to minimize differences due to weight and standardize larval age [last instar larvae are between 180 and $250 \mathrm{mg}$ (Ramarao et al., 2012)]. Before injecting larvae with the pathogen, we stored them at $4^{\circ} \mathrm{C}$ overnight to induce torpidity, which renders them immobile and makes them easier to inject. We injected $10 \mu \mathrm{l}$ of varying doses of $B$. anthracis into the posterior cuticle of each larva and then allowed them to recover for $15-30 \mathrm{~min}$ at room temperature before placing them at $37^{\circ} \mathrm{C}$ and monitoring larval survival every $24 \mathrm{~h}$. Larval death is easy to assess as they turn black shortly before death due to melanization (Tsai et al., 2016). Under these conditions, we find that B. anthracis Sterne kills G. mellonella in a dose-dependent manner (Figure 1A).

Our next step was to determine whether the survival rate of $G$. mellonella differed when injected with the wild-type $B$. anthracis Sterne or an attenuated mutant. We used the $\Delta \mathrm{pXO} 1$ mutant as this strain lacks the pXO1 plasmid, which encodes the lethal and edema toxins, and is avirulent in mice (van Sorge et al., 2008; Levy et al., 2012). We injected a dose of $\sim 5 \times 10^{4}$ cfu per larva, which was mid-way between the two highest doses in our dose curve. This resulted in an overall survival rate around 20\% when larvae were infected with wild-type B. anthracis Sterne (Figure 1B). In contrast, $70 \%$ of the larvae injected with the attenuated $\Delta \mathrm{pXO} 1$ mutant survived, which was almost identical to the PBS-injected control larvae. We thus find that, consistent with the mammalian model, the $\Delta$ pXO1 mutant is severely attenuated for virulence in G. mellonella.

Although this was promising, we wanted to assess the ability of the G. mellonella model to discern changes in virulence beyond the $\triangle \mathrm{pXO} 1$ mutant. The $\mathrm{pXO} 1$ plasmid encodes as many as 143 open reading frames, including the toxins, and it also plays a role in regulating chromosomal genes (Okinaka et al., 1999; Perego and Hoch, 2008). Therefore, its loss should have profound consequences on the virulence of $B$. anthracis. We wanted to assess whether less severe mutations would also demonstrate attenuated virulence in our G. mellonella infection model. We tested five mutant strains of $B$. anthracis Sterne with disruptions in chromosomal genes that had been previously implicated in virulence (Table 2). These chromosomal genes include $c l p X$, an ATPase that functions as part of the ClpXP protease, and $y c e G H$, which encodes the last two genes of a 6-gene cluster of tellurite resistance genes. Our lab has previously linked these genes to defense against AMPs $(c l p X)$ and ROS $(y c e G H)$ (McGillivray et al., 2009; Franks et al., 2014). We also looked at purH, which is critical for purine biosynthesis (Jenkins et al., 2011) along with $m n t A$, part of a manganese transport system, and $h t r A$, a serine protease, which are both important for defenses against ROS (Gat et al., 2005; Chitlaru et al., 2011). Four of these genes, clpX, purH, $m n t A$, and $h \operatorname{tr} A$, had been constructed in a fullyvirulent strain of $B$. anthracis $\left(\mathrm{pXO}^{+}, \mathrm{pXO} 2^{+}\right)$and shown to be attenuated in mammalian infection models so we were confident these represented true virulence determinants of $B$. anthracis. Mutations in $c l p X$ and $y c e G H$ already existed in our lab, and we reconstructed mutations in the other three genes in the Sterne strain. After disrupting purH, mntA and htrA using insertional mutagenesis, we confirmed that our reconstructed mutants had similar in vitro phenotypes as were previously published (Gat et al., 2005; Chitlaru et al., 2011; Jenkins et al., 2011). This included growth deficits in minimal R-medium for $\Delta p u r H$, increased susceptibility to $\mathrm{H}_{2} \mathrm{O}_{2}$ for $\Delta m n t A$ and $\Delta h t r A$ and decreased growth at high temperature for $\triangle h t r A$ (Figure 2). We injected each $G$. mellonella larva with $\sim 5 \times 10^{4} \mathrm{cfu}$ of either the parental (wild-type) or mutant bacteria or a PBS control and found that all of the mutant strains with the exception of $\triangle y c e G H$ exhibited decreased virulence relative to the wild-type strain (Figure 3).

\section{Competition Assay Using G. mellonella}

In our earlier study with the $\Delta y c e G H$ mutant, we saw decreased fitness in mice using a competition model of infection in which wild-type and the $\Delta y c e G H$ strain were mixed at a $1: 1$ ratio and injected as a mixed culture into each mouse to determine relative survival of each bacterial strain (Franks et al., 2014). To develop a comparable competition model in G. mellonella, we mixed wildtype and mutant $B$. anthracis Sterne (either $\Delta h \operatorname{tr} A$ or $\Delta y c e G H$ ) at a 1:1 ratio. Ten microliters of the mixed culture $\left(\sim 1 \times 10^{5}\right.$ total $\mathrm{cfu}$ ) were injected into each larva and the larvae were then incubated for $6 \mathrm{~h}$ at $37^{\circ} \mathrm{C}$. We chose $6 \mathrm{~h}$ as this was long enough for the infection to become established but short enough to preclude any larval mortality. Larvae were then rinsed in $70 \%$ ethanol followed by sterile water to help minimize contamination by surface bacteria before being homogenized in PBS via bead beating. Homogenates were plated on $\mathrm{BHI}$ and $\mathrm{BHI}$-antibiotic plates (Erm5 for $\triangle h t r A$ and Kan50 for $\Delta y c e G H$ ) and the cfu recovered for each strain was calculated. Both the $\triangle h t r A$ and $\triangle y c e G H$ mutants showed decreased fitness in this model with only about $10 \%$ of the recovered cfu belonging to the mutant strains (Figure 4). As a control to make sure that homogenization did not impact relative bacterial survival, we bead-beated and plated a portion of the initial mixed culture prior to injection 
TABLE 2 | Chromosomal virulence mutants used in this study.

\begin{tabular}{|c|c|c|c|c|}
\hline Gene & Function & Phenotype of mutant & $\begin{array}{l}\text { B. anthracis strain used in } \\
\text { mammlian infection models }\end{array}$ & $\begin{array}{l}\text { Derivation of strain } \\
\text { used in current study }\end{array}$ \\
\hline$c / p X$ & $\begin{array}{l}\text { ATPase that functions as } \\
\text { part of CIpXP protease }\end{array}$ & AMP susceptibility & 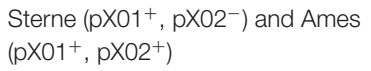 & $\begin{array}{l}\text { Constructed in } \\
\text { McGillivray et al. (2009) }\end{array}$ \\
\hline yceGH & Tellurium resistance & ROS susceptibility & $\begin{array}{l}\text { Sterne } \\
\left(\mathrm{pX01+}, \mathrm{pX02^{- }}\right)\end{array}$ & $\begin{array}{l}\text { Constructed in Franks } \\
\text { et al. (2014) }\end{array}$ \\
\hline purH & Purine biosynthesis & Nutritional deficits & $\begin{array}{l}\text { Ames } \\
\left(\mathrm{pX01+}, \mathrm{pX02}{ }^{+}\right)\end{array}$ & $\begin{array}{l}\text { Reconstructed in this } \\
\text { study }\end{array}$ \\
\hline$m n t A$ & Transporter & ROS susceptibility & $\begin{array}{l}\text { Vollum } \\
\left(\mathrm{pX01+}, \mathrm{pX02}{ }^{+}\right)\end{array}$ & $\begin{array}{l}\text { Reconstructed in this } \\
\text { study }\end{array}$ \\
\hline htrA & Protease & $\begin{array}{l}\text { ROS susceptibility and } \\
\text { growth deficits }\end{array}$ & $\begin{array}{l}\text { Vollum } \\
\left(\mathrm{pX01}+\mathrm{pX02}{ }^{+}\right)\end{array}$ & $\begin{array}{l}\text { Reconstructed in this } \\
\text { study }\end{array}$ \\
\hline
\end{tabular}
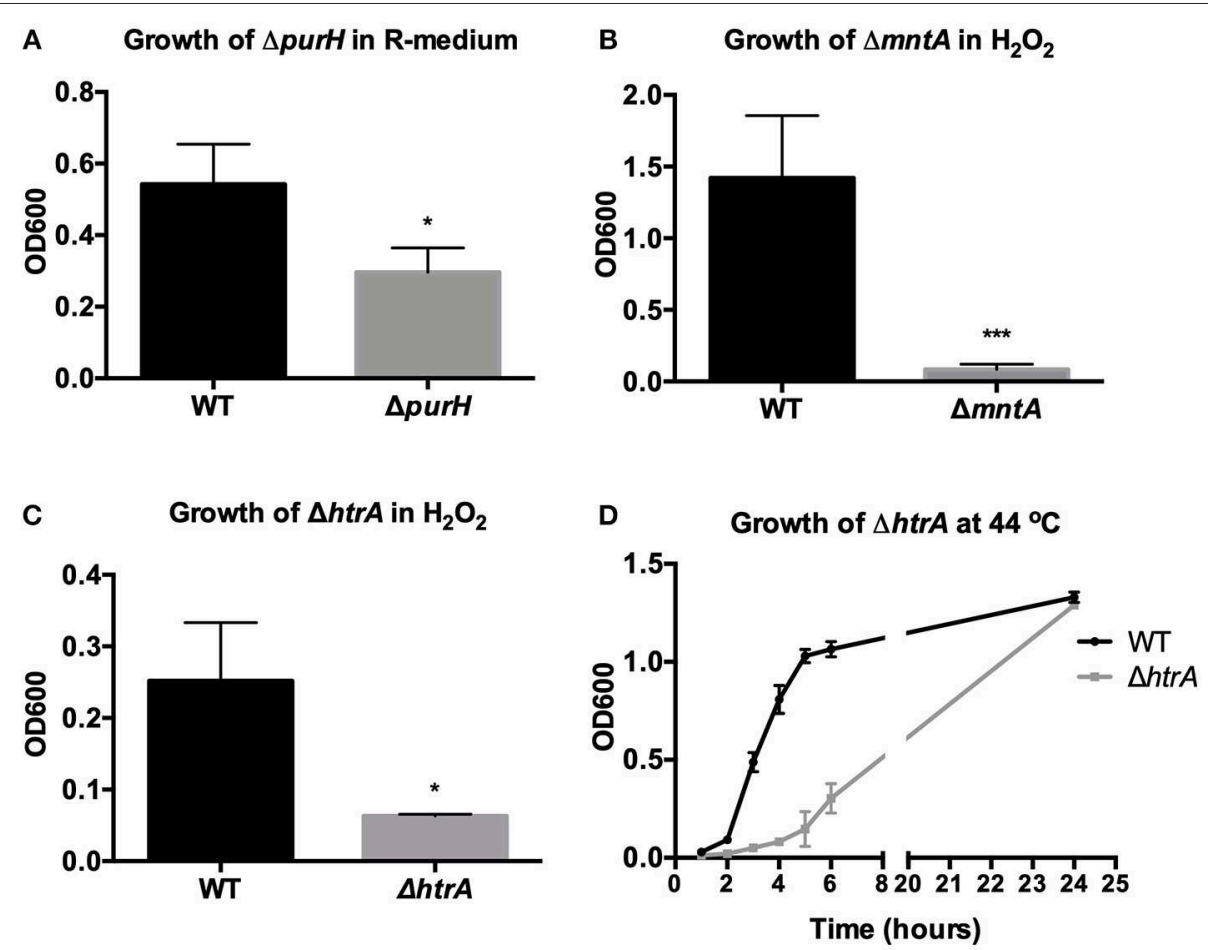

FIGURE 2 | Validation of in vitro phenotype of reconstructed virulence mutants. Growth of wild-type B. anthracis Sterne (WT) was compared to the growth of (A) $\Delta$ purH in minimal R-medium after $24 \mathrm{~h}$ (B) $\Delta m n t A$ in $\mathrm{H}_{2} \mathrm{O}_{2}$ after $24 \mathrm{~h}$ (C) $\Delta h$ trA in $\mathrm{H}_{2} \mathrm{O}_{2}$ after $8 \mathrm{~h}$ and (D) $\Delta h t r A$ at $44^{\circ} \mathrm{C}$ in $\mathrm{BH}$. Assays were repeated three independent times for (A,C,D) and four independent times for $(\mathbf{B})$ and are presented as mean \pm SD. ${ }^{*} p<0.05$ and ${ }^{\star * *} p<0.001$ by unpaired Student's $t$-test.

in the larva and saw no difference in relative survival between the wild-type and mutant strains (data not shown). We conclude that a competition model is also a viable infection model for G. mellonella and may be more sensitive in discerning relative differences in strain fitness than the survival assay.

\section{Use of G. mellonella to Assess Virulence}

Our next goal was to determine whether $G$. mellonella could be used to assess virulence in transposon mutants that displayed promising in vitro phenotypes. Previously, we had conducted several in vitro screens using a library of 5,000 transposon mutants. These screens had yielded a total of 44 mutants that either had altered hemolytic activity, were less virulent in the nematode Caenorhabditis elegans, or had decreased proteolytic activity as evidenced by their inability to degrade casein in 5\% milk plates (McGillivray et al., 2009; Franks et al., 2014); and unpublished data). Two transposon mutants pulled from these screens ultimately led to our characterization of the role of clpX (McGillivray et al., 2009) and yceGH in virulence (Franks et al., 2014), but this left 42 mutants with potentially interesting phenotypes to be investigated. In order to narrow this number down further, we screened these 42 mutants for susceptibility to hydrogen peroxide. $\mathrm{H}_{2} \mathrm{O}_{2}$ is one of the many ROS that are produced by the innate immune system and serve as a critical defense against bacterial pathogens (Lambeth, 2004). Four of these transposon mutants, 2A5, 51D11, 4D5, and 6D10, 

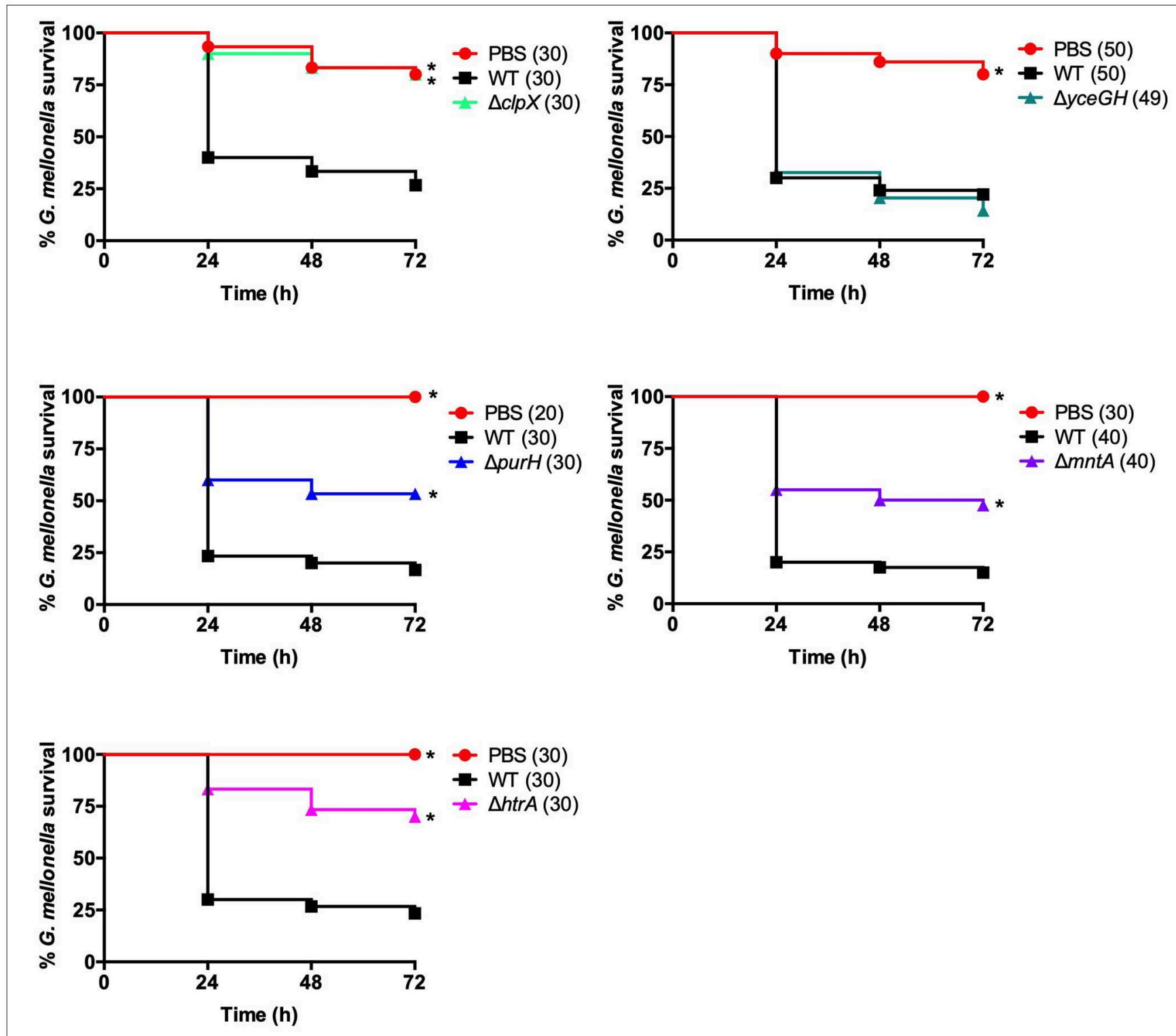

FIGURE 3 | Mutants have attenuated virulence in G. mellonella. Survival of larvae injected with PBS, $\sim 5 \times 10^{4}$ cfu of wild-type B. anthracis Sterne (WT), or the indicated mutant. Each infection was repeated at least three independent times and total number of larvae for each condition are indicated in parentheses. *Indicates statistically different survival than wild-type, $p<0.025$ by the Mantel-Cox log rank test.

were consistently attenuated in their ability to grow in the presence $\mathrm{H}_{2} \mathrm{O}_{2}$ (Figure 5), although there was no difference in growth in plain BHI (data not shown). We next tested these 4 mutants in our G. mellonella survival model. Only one of these mutants, 4D5, was attenuated for virulence relative to the wildtype strain (Figure 6). We determined the site of transposon insertion for $4 \mathrm{D} 5$ and found it disrupted the $a s b C$ gene of the petrobactin biosynthesis operon asbABCDEF. Notably, loss of this operon has been previously linked to both reduced virulence in mice (Cendrowski et al., 2004) and diminished defense against oxidative stress (Hagan et al., 2018). Therefore, we conclude that G. mellonella can effectively identify attenuated virulence mutants of $B$. anthracis Sterne.

\section{DISCUSSION}

A major goal in our development of a $G$. mellonella infection model is to use it to identify novel virulence genes in $B$. anthracis Sterne. This in turn could help identify promising drug targets as part of an antimicrobial strategy aimed at disarming pathogens (Clatworthy et al., 2007). A common strategy for the identification of novel virulence factors is to screen a library of several thousand genetic mutants that cover the entire bacterial genome. In vitro screens are relatively easy to carry out in a high throughput manner, but if too many hits are returned it can be challenging to decide which ones to further characterize, particularly when the mutations are in hypothetical proteins or 

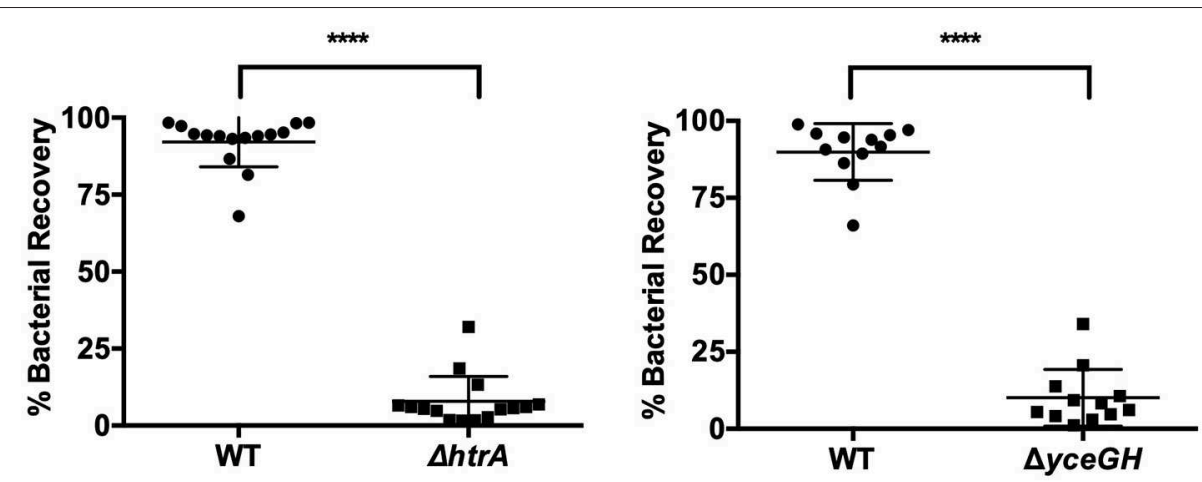

FIGURE 4 | Attenuated virulence is seen in a competition model of infection. Relative survival of wild-type B. anthracis Sterne (WT) and either $\Delta$ htrA (left) or $\Delta y c e G H$ (right) after being mixed at a 1:1 ratio and injected into each larva. Assays were repeated three independent times and all data were combined with each point representing an individual larva. ${ }^{\star * \star *} p<0.0001$ by unpaired Student's $t$-test. Line represents mean \pm SD.

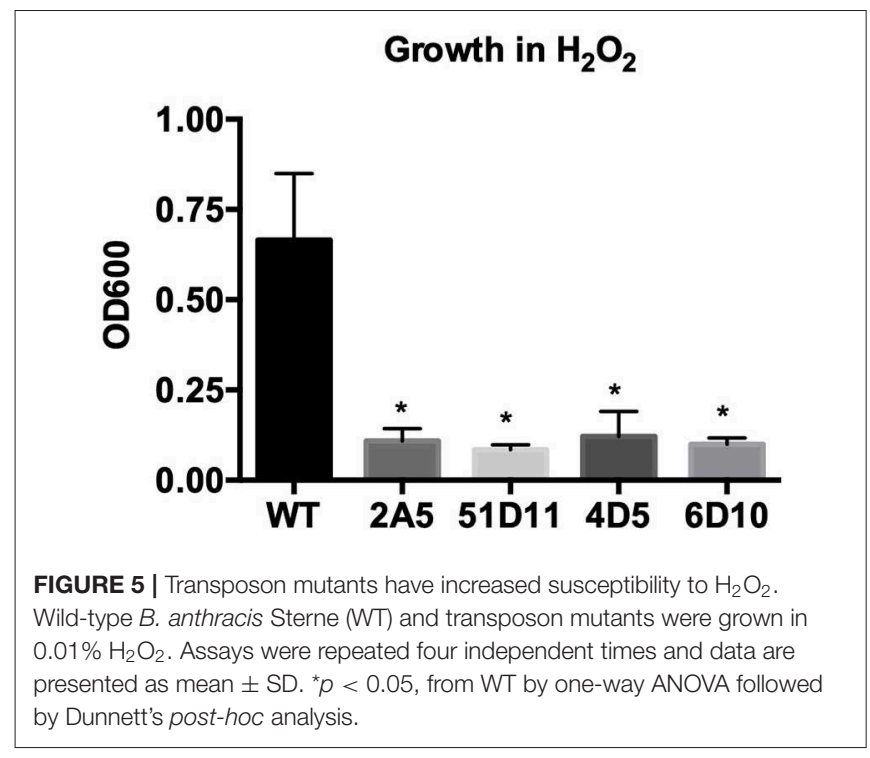

genes of unknown function. The ability to first conduct an in vitro screen and then pass any hits through a practical in vivo infection model would help prioritize mutants for follow-up studies. We have applied this strategy by screening a small pool of transposon mutants first in vitro using $\mathrm{H}_{2} \mathrm{O}_{2}$ and then in vivo in G. mellonella. One of these mutants, 4D5, showed attenuation in both screens. This mutant had a disruption of the petrobactin biosynthetic operon asbABCDEF, which has been previously linked to both resistance to oxidative stress (Hagan et al., 2018) and virulence in a mouse model of infection (Cendrowski et al., 2004). While this is not a novel virulence determinant, it confirms that this strategy can effectively identify a virulence mutant of $B$. anthracis in an unbiased manner.

We developed two different G. mellonella infection models to assess virulence. Both the survival and competition assays were effective as each discerned attenuated virulence in $B$. anthracis Sterne using known virulence mutants. Of the two, the competition assay is likely more sensitive in detecting changes in

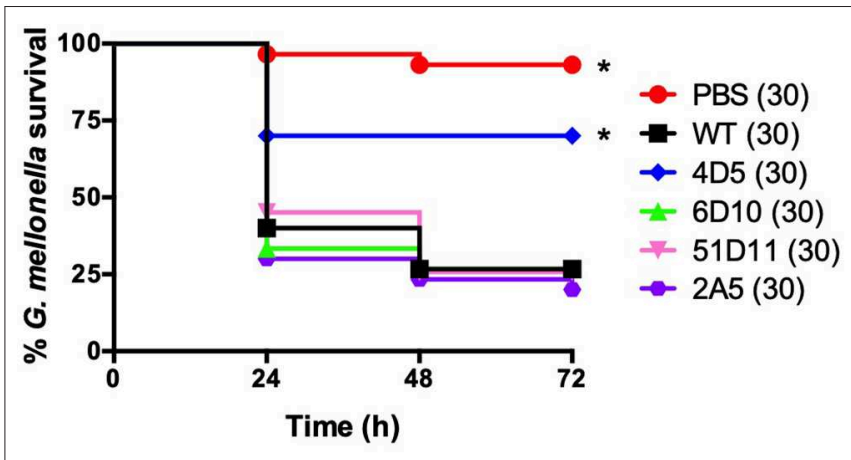

FIGURE 6 | The 4D5 transposon mutant has decreased virulence in G. mellonella. Survival of larvae injected with PBS or $\sim 5 \times 10^{4}$ cfu of wild-type $B$. anthracis Sterne (WT) or the indicated transposon mutant. Each infection was repeated at least three independent times and total number of larvae for each condition are indicated in parentheses. *Indicates statistically different survival than wild-type, $p<0.01$ by the Mantel-Cox log rank test.

bacterial fitness, as we were able to see decreased virulence of the $\triangle y c e G H$ mutant using this assay but not with the survival assay. A recent study established a health index scoring system for $G$. mellonella that, in addition to survival, assesses activity, cocoon formation and melanization; using these additional metrics may increase the sensitivity of the assay rather than relying on survival as the sole data point (Loh et al., 2013). Even so, we chose to use the survival assay when assessing the virulence of our transposon mutants in order to focus on mutants with a strong phenotype.

We obtained G. mellonella larvae from an online bait shop. This was easy and cost-effective, but allowed no control over the age, rearing conditions or genetic background of the $G$. mellonella we received. This could lead to variability from assay to assay even when using a standardized larval weight. In the $G$. mellonella survival assays we performed for this study, injection of $\sim 5 \times 10^{4} \mathrm{cfu}$ of wild-type $B$. anthracis Sterne resulted in a median larval survival rate of $26.3 \%$ with a standard deviation of $14.5 \%$ for a total of 160 worms over 16 independent experiments. 
Therefore, despite some variability in survival rates, the assay was consistent enough to be reliable. In order to make the larvae easier to inject, we stored them at $4^{\circ} \mathrm{C}$ to induce a torpid state prior to handling. An earlier study found that exposure of $G$. mellonella larvae to temperatures of $4^{\circ} \mathrm{C}$ before infection with Candida albicans resulted in increased larval survival, which may have been mediated by increased expression of AMPs and increased hemocyte density at the lower temperature (Mowlds and Kavanagh, 2008). Thus, it is possible the exposure to decreased temperature could have affected the larval response to infection. Regardless, since all of our larvae were treated in the same manner, our results should remain comparatively valid. We will note that even when the larvae were immobile, we still had some injection-induced trauma. This is can be seen when comparing the $98 \%$ survival rate in our uninjected controls across all of our assays (100 larvae total) to the $87 \%$ survival rate in our PBS-injected control groups (180 larvae total). However, a survival rate of $<80 \%$ in the PBS-injected group was rare, occurring in only two of our assays, and is likely indicative of poor injection technique or underlying health issues in the larvae and should be taken into consideration, especially if it is a recurring issue.

There are a number of advantages to using an invertebrate infection model including reduced infrastructure needs and fewer regulatory requirements. We find that $G$. mellonella is a promising animal model for $B$. anthracis Sterne with results that correlate well with those in mammalian models of infection. We also see a number of advantages with the G. mellonella model. The larvae are large enough to be directly injected allowing for precise pathogen exposure and more consistent results. G. mellonella can also be incubated at $37^{\circ} \mathrm{C}$, which is important for temperature-sensitive gene expression in bacterial pathogens (Shapiro and Cowen, 2012). However, there are also some drawbacks. Unlike C. elegans, G. mellonella does not offer an ingestion route of infection, as a prior attempt to orally infect G. mellonella with B. anthracis Sterne proved unsuccessful

\section{REFERENCES}

Cendrowski, S., MacArthur, W., and Hanna, P. (2004). Bacillus anthracis requires siderophore biosynthesis for growth in macrophages and mouse virulence. Mol. Microbiol. 51, 407-417. doi: 10.1046/j.1365-2958.2003.03861.x

Chitlaru, T., Zaide, G., Ehrlich, S., Inbar, I., Cohen, O., and Shafferman, A. (2011). $\mathrm{HtrA}$ is a major virulence determinant of Bacillus anthracis. Mol Microbiol. 81, 1542-1559. doi: 10.1111/j.1365-2958.2011.07790.x

Clatworthy, A. E., Pierson, E., and Hung, D. T. (2007). Targeting virulence: a new paradigm for antimicrobial therapy. Nat. Chem. Biol. 3, 541-548. doi: $10.1038 /$ nchembio.2007.24

Fedhila, S., Buisson, C., Dussurget, O., Serror, P., Glomski, I. J., Liehl, P., et al. (2010). Comparative analysis of the virulence of invertebrate and mammalian pathogenic bacteria in the oral insect infection model Galleria mellonella. J. Invertebr. Pathol. 103, 24-29. doi: 10.1016/j.jip.2009.09.005

Franks, S. E., Ebrahimi, C., Hollands, A., Okumura, C. Y., Aroian, R. V., Nizet, V., et al. (2014). Novel role for the yceGH tellurite resistance genes in the pathogenesis of Bacillus anthracis. Infect. Immun. 82, 1132-1140. doi: 10.1128/IAI.01614-13

Gat, O., Mendelson, I., Chitlaru, T., Ariel, N., Altboum, Z., Levy, H., et al. (2005). The solute-binding component of a putative $\mathrm{Mn}(\mathrm{II}) \mathrm{ABC}$ transporter (MntA)
(Fedhila et al., 2010). There are also limited genetic tools in $G$. mellonella making it difficult to manipulate the host side of the infection model, in contrast to C. elegans where RNA-mediated interference is a well-established technique for knocking down host gene expression (Glavis-Bloom et al., 2012). Therefore, we see $G$. mellonella as a useful complement to the other established infection models, both vertebrate and invertebrate, that currently exist for $B$. anthracis. Ultimately, we hope the development of the G. mellonella infection model will allow us to expand our understanding of $B$. anthracis virulence mechanisms while decreasing our reliance on mammalian models of infection.

\section{DATA AVAILABILITY STATEMENT}

The raw data underlying all figures presented in this publication can be found at https://doi.org/10.6084/m9.figshare.c.4670009.

\section{AUTHOR CONTRIBUTIONS}

SM conceived of the study, designed the experiments, and wrote the manuscript. JM and MR performed the experiments and also contributed to data analysis, experimental design, and manuscript editing.

\section{FUNDING}

This work was supported by a grant from the TCU Research and Creative Activities Fund to SM and a grant from the TCU College of Science and Engineering Research Center to MR. The TCU Mary Couts Burnett library provided funding for open access fees associated with this publication.

\section{ACKNOWLEDGMENTS}

We thank Dr. Mark Demarest for helpful comments on the unpublished manuscript. is a novel Bacillus anthracis virulence determinant. Mol. Microbiol. 58, 533-551. doi: 10.1111/j.1365-2958.2005.04848.x

Glavis-Bloom, J., Muhammed, M., and Mylonakis, E. (2012). Of model hosts and man: using Caenorhabditis elegans, Drosophila melanogaster and Galleria mellonella as model hosts for infectious disease research. Adv. Exp Med Biol. 710, 11-17. doi: 10.1007/978-1-4419-5638-5_2

Gravato-Nobre, M. J., and Hodgkin, J. (2005). Caenorhabditis elegans as a model for innate immunity to pathogens. Cell Microbiol. 7, 741-751. doi: 10.1111/j.1462-5822.2005. 00523.x

Hagan, A. K., Plotnick, Y. M., Dingle, R. E., Mendel, Z. I., Cendrowski, S. R., Sherman, D. H., et al. (2018). Petrobactin protects against oxidative stress and enhances sporulation efficiency in Bacillus anthracis sterne. MBio. 9:e02079-18. doi: 10.1128/mBio.02079-18

Harvill, E. T., Lee, G., Grippe, V. K., and Merkel, T. J. (2005). Complement depletion renders C57BL/6 mice sensitive to the Bacillus anthracis Sterne strain. Infect. Immun. 73, 4420-4422. doi: 10.1128/IAI.73.7.4420-442 2.2005

Jenkins, A., Cote, C., Twenhafel, N., Merkel, T., Bozue, J., and Welkos, S. (2011). Role of purine biosynthesis in Bacillus anthracis pathogenesis and virulence. Infect. Immun. 79, 153-166. doi: 10.1128/IAI.00925-10 
Kavanagh, K., and Reeves, E. P. (2004). Exploiting the potential of insects for in vivo pathogenicity testing of microbial pathogens. FEMS Microbiol. Rev. 28, 101-112. doi: 10.1016/j.femsre.2003.09.002

Kern, J., and Schneewind, O. (2010). BslA, the S-layer adhesin of B. anthracis, is a virulence factor for anthrax pathogenesis. Mol. Microbiol. 75, 324-332. doi: 10.1111/j.1365-2958.2009.06958.x

Kho, M. F., Bellier, A., Balasubramani, V., Hu, Y., Hsu, W., NielsenLeRoux, C., et al. (2011). The pore-forming protein Cry5B elicits the pathogenicity of Bacillus sp. against Caenorhabditis elegans. PLoS ONE 6:e29122. doi: 10.1371/journal.pone.0029122

Koehler, T. M., Dai, Z., and Kaufman-Yarbray, M. (1994). Regulation of the Bacillus anthracis protective antigen gene: $\mathrm{CO}_{2}$ and a trans-acting element activate transcription from one of two promoters. J. Bacteriol. 176, 586-595. doi: 10.1128/jb.176.3.586-595.1994

Kwon, Y. M., and Ricke, S. C. (2000). Efficient amplification of multiple transposon-flanking sequences. J. Microbiol. Methods 41, 195-199. doi: 10.1016/S0167-7012(00)00159-7

Lambeth, J. D. (2004). NOX enzymes and the biology of reactive oxygen. Nat. Rev. Immunol. 4, 181-189. doi: 10.1038/nri1312

Levy, H., Weiss, S., Altboum, Z., Schlomovitz, J., Glinert, I., Sittner, A., et al. (2012). Differential contribution of Bacillus anthracis toxins to pathogenicity in two animal models. Infect. Immun. 80, 2623-2631. doi: 10.1128/IAI.0 0244-12

Loh, J. M., Adenwalla, N., Wiles, S., and Proft, T. (2013). Galleria mellonella larvae as an infection model for group A streptococcus. Virulence 4, 419-428. doi: $10.4161 /$ viru. 24930

McGillivray, S. M., Ebrahimi, C. M., Fisher, N., Sabet, M., Zhang, D. X., Chen, Y., et al. (2009). ClpX contributes to innate defense peptide resistance and virulence phenotypes of Bacillus anthracis. J. Innate Immun. 1, 494-506. doi: 10.1159/000225955

Mowlds, P., and Kavanagh, K. (2008). Effect of pre-incubation temperature on susceptibility of Galleria mellonella larvae to infection by Candida albicans. Mycopathologia 165, 5-12. doi: 10.1007/s11046-0079069-9

Okinaka, R., Cloud, K., Hampton, O., Hoffmaster, A., Hill, K., Keim, P., et al. (1999). Sequence, assembly and analysis of pX01 and pX02. J. Appl. Microbiol. 87, 261-262. doi: 10.1046/j.1365-2672.1999.00883.x
Perego, M., and Hoch, J. A. (2008). Commingling regulatory systems following acquisition of virulence plasmids by Bacillus anthracis. Trends Microbiol. 16, 215-221. doi: 10.1016/j.tim.2008.01.010

Ramarao, N., Nielsen-Leroux, C., and Lereclus, D. (2012). The insect Galleria mellonella as a powerful infection model to investigate bacterial pathogenesis. J. Vis. Exp. 70:e4392. doi: 10.3791/4392

Read, T. D., Peterson, S. N., Tourasse, N., Baillie, L. W., Paulsen, I. T., Nelson, K. E., et al. (2003). The genome sequence of Bacillus anthracis Ames and comparison to closely related bacteria. Nature $423,81-86$. doi: $10.1038 /$ nature 01586

Ristroph, J. D., and Ivins, B. E. (1983). Elaboration of Bacillus anthracis antigens in a new, defined culture medium. Infect. Immun. 39, 483-486. doi: 10.21236/ADA116827

Shapiro, R. S., and Cowen, L. E. (2012). Thermal control of microbial development and virulence: molecular mechanisms of microbial temperature sensing. MBio 3:e00238-12. doi: 10.1128/mBio.00238-12

Tsai, C. J., Loh, J. M., and Proft, T. (2016). Galleria mellonella infection models for the study of bacterial diseases and for antimicrobial drug testing. Virulence 7, 214-229. doi: 10.1080/21505594.2015.1135289

van Sorge, N. M., Ebrahimi, C. M., McGillivray, S. M., Quach, D., Sabet, M., Guiney, D. G., et al. (2008). Anthrax toxins inhibit neutrophil signaling pathways in brain endothelium and contribute to the pathogenesis of meningitis. PLoS ONE 3:e2964. doi: 10.1371/journal.pone.0002964

Welkos, S. L., and Friedlander, A. M. (1988). Pathogenesis and genetic control of resistance to the Sterne strain of Bacillus anthracis. Microb. Pathog. 4, 53-69. doi: 10.1016/0882-4010(88)90048-4

Conflict of Interest: The authors declare that the research was conducted in the absence of any commercial or financial relationships that could be construed as a potential conflict of interest.

Copyright (C) 2019 Malmquist, Rogan and McGillivray. This is an open-access article distributed under the terms of the Creative Commons Attribution License (CC BY). The use, distribution or reproduction in other forums is permitted, provided the original author(s) and the copyright owner(s) are credited and that the original publication in this journal is cited, in accordance with accepted academic practice. No use, distribution or reproduction is permitted which does not comply with these terms. 